\title{
Increased P-Wave Duration and P-Wave Dispersion in Patients with Aortic Stenosis
}

\author{
Hasan Turhan, M.D., ${ }^{*}$ Ertan Yetkin, M.D., † Ramazan Atak, M.D.,* \\ Tayfun Altinok, M.D., † Kubilay Senen, M.D.,* Mehmet Ileri, M.D.,** \\ Hatice Sasmaz, M.D., * Sengul Cehreli, M.D., † and Emine Kutuk, M.D.*
}

From the *Turkiye Yuksek Ihtisas Hospital, Department of Cardiology, Ankara, Turkey; and $\dagger$ Department of Cardiology, Medical Faculty, Inonu University, Malatya, Turkey

\begin{abstract}
Background: P-wave dispersion (PWD), defined as the difference between the maximum and minimum P-wave duration, has been proposed as being useful for the prediction of paroxysmal atrial fibrillation (AF). AF is the most common arrhythmia and an important prognostic indicator for clinical deterioration in patients with aortic stenosis (AS). The aim of the present study was to evaluate PWD in patients with AS.

Methods: The study population consisted of two groups: Group I consisted of 98 patients with AS (76 men, 22 women; aged $63 \pm 8$ years) and group II consisted of 98 healthy subjects (same age and sex) without any cardiovascular disease. A 12-lead electrocardiogram was recorded for each subject. The P-wave duration was calculated in all leads of the surface electrocardiogram. The difference between the maximum and minimum P-wave duration was calculated and was defined as the PWD. All patients and control subjects were also evaluated by echocardiography to measure the left atrial diameter, left ventricular ejection fraction, left ventricular wall thicknesses, and the maximum and mean aortic gradients. Patients were also evaluated for the presence of paroxysmal AF.

Results: Maximum P-wave duration and PWD of group I were found to be significantly higher than those of group II. In addition, patients with paroxysmal AF had significantly higher PWD than those without paroxysmal AF. There was no significant difference between the two groups regarding minimum P-wave duration. In addition, there was no significant correlation between echocardiographic variables and PWD.

Conclusion: PWD, indicating increased risk for paroxysmal AF, was found to be significantly higher in patients with AS than in those without it. Further assessment of the clinical utility of PWD for the prediction of paroxysmal AF in patients with severe AS will require longer prospective studies.
\end{abstract}

A.N.E. 2003;8(1):18-21

aortic stenosis; P-wave dispersion; atrial fibrillation

P-wave dispersion (PWD), defined as the difference between the maximum and minimum $\mathrm{P}$-wave $\mathrm{du}$ ration, is a new electrocardiographic marker that has been associated with inhomogeneous and discontinuous propagation of sinus impulses. ${ }^{1,2}$ The correlation between the presence of interatrial and intraatrial conduction abnormalities and the induction of paroxysmal atrial fibrillation $(\mathrm{AF})$ has been well documented. ${ }^{3,4}$ In addition, prolonged P-wave duration and increased PWD are commonly found in patients with a history of paroxysmal $\mathrm{AF}^{2,5,6} \mathrm{AF}$, whether chronic or paroxysmal, is the most common sustained arrhythmia encountered in clinical practice that produces substantial excess cardiovascular morbidity and mortality. ${ }^{7,8}$ The estimation of the probability of a patient developing frequent $\mathrm{AF}$ paroxysms might guide the clinician in the management of paroxysmal AF. Aortic stenosis (AS) is the most common cause of left ventricular outflow tract obstruction. ${ }^{9} \mathrm{AF}$ is the most common arrhythmia seen in patients with severe AS. ${ }^{10}$ Furthermore, it is an important prognostic indicator for clinical deterioration in patients with AS. ${ }^{9}$ 
The aim of the present study was to investigate a new electrocardiographic marker, PWD, in patients with severe AS. To our knowledge, this is the first study on the effects of AS on PWD.

\section{METHODS}

\section{Study Population}

The study population consisted of two groups: Group I consisted of 98 patients with degenerative AS (76 men, 22 women; aged $63 \pm 8$ years) and group II consisted of 98 healthy subjects, same age and sex (76 men, 22 women; aged $62 \pm$ 9 years), without any cardiovascular disease. At the time of electrocardiographic recording, all subjects were in sinus rhythm, and none of them were taking any type of antiarrhythmic agent. Patients who had coronary artery disease, hypertension, moderate to severe aortic regurgitation, more than mild degree of mitral regurgitation, mitral stenosis, diabetes mellitus, hyperthyroidism, pericardial effusion, chronic obstructive pulmonary disease, ventricular preexcitation, atrioventricular conduction abnormalities, or abnormal serum electrolytes were excluded from the study.

\section{Electrocardiographic Measurement}

Twelve-lead electrocardiogram was recorded for each subject at a rate of $50 \mathrm{~mm} / \mathrm{s}$ in the supine position. The electrocardiograms were coded and all annotations were masked. The measurements of the P-wave duration were performed manually by two of the investigators without knowledge of the clinical status of the patients and controls. To improve accuracy, measurements were performed with calipers and magnifying lens for defining the electrocardiographic deflection. P-wave duration was measured from the onset to the offset of the $\mathrm{P}$-wave. The onset and offset of the P-wave were defined as the junction between the P-wave pattern and the isoelectric line. After completion of the measurements, all electrocardiograms were decoded. PWD was defined as the difference between the maximum and minimum P-wave duration. Intraobserver and interobserver coefficients of variation were found to be $4.1 \%$ and $4.4 \%$ for PWD, respectively. All patients were evaluated for the presence of documented paroxysmal AF during their past medical history. Electrocardiographic diagnosis of paroxysmal AF was made according to
Bellet's definition. ${ }^{11}$ Paroxysmal AF was diagnosed in a patient with a history of recurrent episodes of AF lasting more than 2 minutes and less than 7 days.

\section{Echocardiographic Evaluation}

Transthoracic echocardiographic examination was performed for each subject. Maximum and mean aortic gradients were calculated by Doppler studies. Color flow Doppler was used to detect the presence of mitral and aortic regurgitation. Left atrial diameter, left ventricular end-diastolic and end-systolic diameters, left ventricular ejection fraction, and left ventricular wall thicknesses were measured by M mode echocardiography. Pulmonary artery systolic pressure was calculated with the help of continuous-wave Doppler studies using the Bernoulli equation.

\section{Statistical Analysis}

All numeric variables were expressed as mean \pm SD and categorical variables were expressed as percentage. Statistical analysis was performed using unpaired $t$-test, Mann-Whitney $\mathrm{U}$ test and chisquare test where appropriate, and Pearson correlation test was used to determine the correlation between PWD and echocardiographic variables in patients with AS. A P value $<0.05$ was considered statistically significant.

\section{RESULTS}

There was no statistically significant difference between the two groups with respect to age and gender $(\mathrm{P}>0.05)$. Eight patients $(8 \%)$ were diagnosed as having documented paroxysmal AF with the evaluation of their past medical history. Echocardiographic variables were similar in patients with and without paroxysmal AF (Table 1). Maximum P-wave duration and PWD of group I were found to be significantly higher than those of group II $(\mathrm{P}<0.0001$, Table 2). However, there was no statistically significant difference between group I and group II regarding minimum P-wave duration $(\mathrm{P}>0.05$, Table 2). Patients with AS and paroxysmal AF had higher maximum $\mathrm{P}$-wave duration and $\mathrm{PWD}$ than those without paroxysmal AF $(\mathrm{P}<0.001$, Table 2). There was no statistically significant difference between the values of minimum P-wave duration in patients with and without paroxysmal $\mathrm{AF}(\mathrm{P}>0.05$, Table 2$)$. We found no significant 
Table 1. Echocardiographic Variables of Patients with and without Paroxysmal Atrial Fibrillation

\begin{tabular}{lccr}
\hline Variable & $\begin{array}{c}\text { Patients with } \\
\text { PAF }(\mathbf{n}=\mathbf{8})\end{array}$ & $\begin{array}{c}\text { Patients without } \\
\text { PAF }(\mathbf{n}=\mathbf{9 0})\end{array}$ & P \\
\hline Maximum aortic gradient (mmHg) & $82 \pm 7$ & $78 \pm 6$ & NS \\
Mean aortic gradient (mmHg) & $43 \pm 6$ & $42 \pm 7$ & NS \\
Thickness of interventricular septum (cm) & $1.43 \pm 0.12$ & $1.38 \pm 0.14$ & NS \\
Thickness of posterior wall (cm) & $1.34 \pm 0.14$ & $1.32 \pm 0.09$ & NS \\
Left atrial diameter (cm) & $3.8 \pm 0.52$ & $3.8 \pm 0.65$ & NS \\
Left ventricular ejection fraction (\%) & $66 \pm 5$ & $66 \pm 6$ & NS \\
\hline
\end{tabular}

NS: Nonsignificant; PAF: Paroxysmal atrial fibrillation.

correlation between PWD and echocardiographic variables including left atrial diameter, maximum and mean aortic gradients, left ventricular posterior wall, and interventricular septal thicknesses and left ventricular ejection fraction $(\mathrm{P}>0.05$ for all).

\section{DISCUSSION}

In this study, we found that patients with AS have longer maximum P-wave duration and higher PWD than do healthy control subjects. Besides, patients with AS and paroxysmal AF have higher values of maximum P-wave duration and PWD than those without paroxysmal AF. An interesting finding of this study is that there is no significant correlation between PWD and echocardiographic variables.

PWD is a new electrocardiographic marker that has been associated with the inhomogeneous and discontinuous propagation of sinus impulses., ${ }^{1,2}$ Prolongation of intraatrial and interatrial conduction time and inhomogeneous propagation of sinus impulses are well-known electrophysiological characteristics in patients with paroxysmal AF. ${ }^{1,2}$

Table 2. Electrocardiographic Variables in Patients with Aortic Stenosis and in Healthy Control Subjects

\begin{tabular}{lrrr}
\hline & \multicolumn{2}{c}{$\begin{array}{c}\text { Patients with } \\
\text { Vortic Stenosis }\end{array}$} \\
\cline { 3 - 4 } Variable & $\begin{array}{c}\text { Control } \\
\text { Subjects }\end{array}$ & PAF (-) & PAF (+) \\
\hline P minimum (ms) & $76 \pm 8$ & $78 \pm 8^{\mathrm{a}}$ & $78 \pm 7^{\mathrm{a}}$ \\
$\mathrm{P}$ maximum (ms) & $108 \pm 7$ & $121 \pm 8^{\mathrm{b}, \mathrm{c}}$ & $130 \pm 8^{\mathrm{b}}$ \\
$\begin{array}{l}\text { P-wave } \\
\text { dispersion (ms) }\end{array}$ & $32 \pm 5$ & $43 \pm 5^{\mathrm{b}, \mathrm{c}}$ & $52 \pm 5^{\mathrm{b}}$ \\
\hline
\end{tabular}

${ }^{a} \mathrm{P}>0.05$ versus control subjects, ${ }^{\mathrm{b}} \mathrm{P}<0.0001$ versus control subjects, ${ }^{\mathrm{C}} \mathrm{P}<0.001$ versus patients with PAF. PAF: Paroxysmal atrial fibrillation; P minimum: Minimum P-wave duration; P maximum: Maximum P-wave duration.
Moreover, the correlation between the presence of intraatrial conduction abnormalities and the induction of paroxysmal AF has been well documented. ${ }^{3,12}$ This electrophysiological characteristic results in increased PWD on electrocardiographic measurements. Therefore, PWD can be used to separate patients with a high risk of AF during sinus rhythm. ${ }^{12}$

Degenerative calcific AS is now the most common cause of left ventricular outflow tract obstruction in adults. ${ }^{9} \mathrm{AF}$ is the most common arrhythmia and an important prognostic indicator for clinical deterioration in patients with AS. ${ }^{9,10} \mathrm{AF}$ in a patient with severe AS results in the loss of the atrial contribution to the left ventricular filling, which causes a precipitous decline in cardiac output and aggravation of symptoms. ${ }^{9}$ The estimation of the probability of a patient developing frequent AF paroxysms might guide the clinician in the management of paroxysmal AF.

P-wave duration and PWD have been reported to be influenced by the autonomic tone, which induces changes in the velocity of impulse propagation. ${ }^{13}$ Furthermore, AF, either intermittent or chronic, may be influenced by autonomic activity. ${ }^{14}$ Ramirez-Gil et al. ${ }^{15}$ have shown increased sympathetic activity in patients with AS. In addition, Tükek et al. ${ }^{16}$ have reported that increased sympathetic activity causes a significant increase in PWD. As a result of these findings, we can suggest that increased sympathetic activity may be the underlying cause of higher PWD in patients with severe AS.

We found no significant correlation between PWD and echocardiographic variables (left atrial diameter, left ventricular ejection fraction, thickness of interventricular septum and posterior wall, maximum and mean aortic gradients). In addition, patients with and without paroxysmal AF had 
similar echocardiographic variables (Table 1). Earlier, some authors have suggested that left atrial dimension, ${ }^{17-20}$ left ventricular dysfunction, ${ }^{17}$ and the presence of organic heart disease ${ }^{21}$ could predict paroxysmal AF. However, our patients had a preserved left ventricular function, and a more or less normal mean left atrial maximal diameter. Recently, we reported that PWD in patients with mitral stenosis is not related to left atrial diameter and the decrease in PWD after PMBV is not correlated with the improvement in left atrial diameter, mitral valve area, mean mitral gradient, and left atrial pressure. ${ }^{22}$ Although some authors ${ }^{16,20}$ reported that left atrial diameter is a significant predictor of AF episodes, some others ${ }^{1,23,24}$ have reported that left atrial maximal diameter is not a significant predictor of paroxysmal AF.

\section{CONCLUSION}

In conclusion, $\mathrm{PWD}$, indicating increased risk for paroxysmal $A F$, is significantly higher in patients with AS than in healthy control subjects. In addition, PWD in patients with AS associated with paroxysmal $\mathrm{AF}$, is significantly higher than in those without paroxysmal AF. Further assessment of the clinical utility of PWD for the prediction of paroxysmal AF in patients with severe AS will require longer prospective studies.

\section{REFERENCES}

1. Dilaveris PE, Gialafos EJ, Andrikopoulos GK, et al. Clinical and electrocardiographic predictors of recurrent atrial fibrillation. Pacing Clin Electrophysiol 2000;23:352-358.

2. Dilaveris PE, Gialafos EJ, Sideris S, et al. Simple electrocardiographic markers for the prediction of paroxysmal idiopathic atrial fibrillation. Am Heart J 1998;135:733738.

3. Buxton $\mathrm{AE}$, Waxman $\mathrm{HL}$, Marchlinski $\mathrm{FE}$, et al. Atrial conduction: effects of extrastimuli with and without atrial dysrhythmias. Am J Cardiol 1984;54:755-761.

4. Leier CV, Meacham JA, Schall SF. Prolonged atrial conduction: A major predisposing factor for atrial flutter. Circulation 1978;57:213-216.

5. Gialafos JE, Dilaveris PE, Gialafos EJ, et al. P-wave dispersion: A valuable electrocardiographic marker for the prediction of paroxysmal lone atrial fibrillation. Ann Noninvasive Electrocardiol 1999;4:39-45.

6. Yamada T, Fukunami M, Shimonagata T, et al. Dispersion of signal-averaged P-wave duration on precordial body surface in patients with paroxysmal atrial fibrillation. Eur Heart J 1999;20:211-220.
7. Kannel WB, Abbott RD, Savage DD, et al. Coronary heart disease and atrial fibrillation: The Framingham Study. Am Heart J 1983;106:389-396.

8. Kannel WB, Wolf PA. Epidemiology of atrial fibrillation. In Falk RH, Podrid PJ (eds.): Atrial Fibrillation. Mechanisms and Management. New York, Raven Press, 1992, pp. 81-92.

9. Braunwald E. Valvular heart disease. In Braunwald Zipes Libby (ed.): Heart Disease: A Textbook of Cardiovascular Medicine, 6th Edition Philadelphia, W.B. Saunders, 2001, pp. $1643-1653$.

10. Rubin DN, Griffin BP. Valvular heart disease. In Topol EJ, Marso SP, Griffin BP (eds.): Manual of Cardiovascular Medicine. Philadelphia, Lippincott/Williams \& Wilkins, 2000, pp. 167-232.

11. Bellet S. Atrial Fibrillation (Clinical Manifestations): Clinical Disorders of the Heartbeat. Philadelphia, PA, Lea and Febiger, 1971, pp.223-233.

12. Aytemir $\mathrm{K}$, Ozer $\mathrm{N}$, Atalar $\mathrm{E}$, et al. $\mathrm{P}$ wave dispersion on 12-lead electrocardiography in patients with paroxysmal atrial fibrillation. Pacing Clin Electrophysiol 2000;23:11091112.

13. Cheema AN, Ahmed MW, Kadish AH, et al. Effects of autonomic stimulation and blockade on signal-averaged P-wave duration. J Am Coll Cardiol 1995;26:497-502.

14. Coumel P. Paroxysmal atrial fibrillation: A disorder of autonomic tone? Eur Heart J 1994;15(Suppl. A):9-16.

15. Ramirez-Gil JF, Drobinski G, Carayon A, et al. Neurohormonal profile in aortic valve stenosis. Arch Mal Coeur Vaiss 1993;86:1001-1007.

16. Tukek T, Akkaya V, Demirel S, et al. Effect of valsalva maneuver on surface electrocardiographic $\mathrm{P}$-wave dispersion in paroxysmal atrial fibrillation. Am J Cardiol 2000;85:896899.

17. Flaker GC, Fletcher KA, Rothbart RM, et al. Clinical and echocardiographic features of intermittent atrial fibrillation that predict recurrent atrial fibrillation. Am J Cardiol 1995;76:355-358.

18. Turitto G, Bandarizadeh B, Salciccioli L, et al. Risk stratification for recurrent tachyarrhythmias in patients with paroxysmal atrial fibrillation and flutter: Role of signal averaged electrocardiogram and echocardiography. Pacing Clin Electrophysiol 1998;21:197-201.

19. Henry WL, Morganroth J, Pearlman AS, et al. Relation between echocardiographically determined left atrial size and atrial fibrillation. Circulation 1976;53:273-279.

20. Kerr CR, Boone J, Connolly SJ, et al. The Canadian registry of atrial fibrillation: A noninterventional follow-up of patients after the first diagnosis of atrial fibrillation. Am J Cardiol 1998;82:82N-85N.

21. Suttorp MJ, Kingma H, Koomen EM, et al. Recurrence of paroxysmal atrial fibrillation or flutter after successful cardioversion in patients with normal left ventricular function. Am J Cardiol 1993;71:710-713.

22. Turhan $H$, Yetkin $E$, Senen $K$, et al. Effects of percutaneous mitral balloon valvuloplasty on p-wave dispersion in patients with mitral stenosis. Am J Cardiol 2002;89:607-609.

23. Josephson ME, Kastor JA, Morganroth J. Electrocardiographic left atrial enlargement: Electrophysiological, echocardiographic and hemodynamic correlates. Am J Cardiol 1977;39:967-971.

24. Ishimoto N, Ito M, Kinoshita M. Signal-averaged P-wave abnormalities and atrial size in patients with and without idiopathic paroxysmal atrial fibrillation. Am Heart J 2000;139:684-689. 